\title{
MAESTRO DE LA CIRUGÍA CHILENA 2013 DR. OSVALDO LLANOS LÓPEZ
}

\author{
Outstanding Chilean surgeon
}

\section{Discurso de Homenaje}

Estos son momentos de alto significado para la Sociedad de Cirujanos de Chile:

Se ha nominado un nuevo Maestro de la Cirugía. Este nombramiento le corresponde a la Sociedad y siendo desde luego una designación muy honrosa para el nominado, debe considerarse también como una responsabilidad mayor para la propia Sociedad: a través de sus maestros lo que hace la Sociedad, en el fondo, es establecer ejemplos que deben servir de inspiración a todos sus miembros e incluso a aquellos que, sin haber ingresado a la Sociedad, ejercen esta especialidad. Debiera ser también fuente de inspiración para colegas de otras especialidades por la evidente razón de que los valores positivos deben compartirse. El maestro es, como lo estamos viviendo hoy, un ejemplo vivo y activo. No es un recuerdo, no es un concepto estático como pudiera serlo una imagen o incluso una estatua, sino alguien que ha hecho y hace de su vida y de su trabajo, una búsqueda incansable de excelencia y que por definición y por decisión no se la guarda para sí sino que la transmite en forma permanente a otros. La transmisión de la excelencia supone entonces un acto de generosidad que hoy queremos reconocer y agradecer en Osvaldo. Por esta vía de alguna manera todos pasamos a ser discípulos de este maestro.

He sido honrado al ser designado responsable de este homenaje, lo que agradezco de manera muy profunda. Sin embargo, a lo largo de mi vida he sido objeto de otro honor que quiero reconocer ahora, $y$ es la larga e inquebrantable amistad que me une al nuevo maestro desde hace ya varias décadas. En lo personal su ejemplo ha sido para mí una especie de reserva moral a la que suelo recurrir, especialmente en momentos difíciles. Sin embargo, trataré de separar este vínculo personal, que creo que ha influido en la decisión del Directorio al designarme para efectuar el homenaje, de la objetividad necesaria para reconocer al nuevo maestro. $\mathrm{Al}$ respecto, se da hoy una situación que creo inédita en nuestra Sociedad. Hace algunos años, ante mi nombramiento de Maestro le correspondió al Dr. Llanos hacer la semblanza. En ese momento hice presente en mi

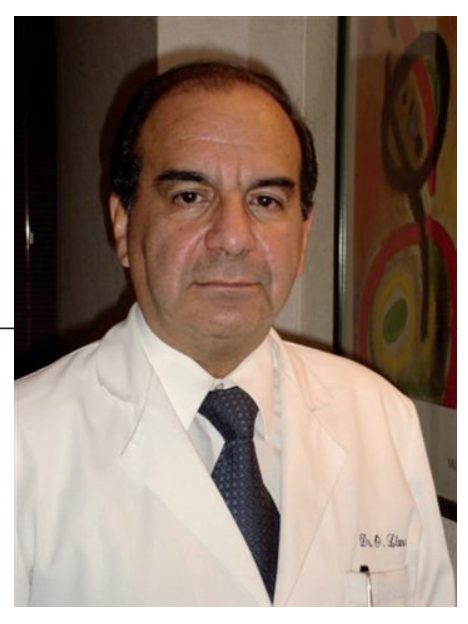

discurso de agradecimiento que con gusto habría invertido los roles porque creo que Osvaldo encarna con creces la persona del Maestro con una idoneidad estimulante y muy difícil de alcanzar. Por lo tanto, humildemente como maestro rindo homenaje al nuevo maestro. Quisiera señalar que se da entre nosotros otra situación, probablemente también inédita: El Dr. Llanos me hizo el año 2001 (hace 12 años) una Gastrectomía Total por un linfoma, y aquí estoy, de modo que además de compañero de trabajo y amigo soy su paciente agradecido. Por esta vía y de manera atípica he aprendido de su maestría lo que es el dumping y las consecuencias de la Y de Roux que desde entonces forma parte de mi anatomía.

No es fácil resumir lo que ha sido la vida de Osvaldo. Aun cuando, como veremos, tiene un extenso Currículum cuya sola lectura justificaría esta nominación, son sus características de hombre íntegro que ha debido convivir con el esfuerzo, con el sacrificio y también con el sufrimiento las que, a mi entender, más la avalan.

Osvaldo nació en Santiago en 1943. Su padre fue Don Osvaldo Llanos Martínez, cuya amplia familia era Colchagüina, y su madre, Doña Olga López Varas era de Copiapó. Tuve el privilegio de conocer a ambos, más a Don Osvaldo: Él trabajó en la empresa CODINA hasta su jubilación. Siempre fue un hombre sencillo, claro en sus valores y conceptos, caballeroso, de actitud modesta y padre orgulloso. Doña Olga fue odontóloga de profesión, celosa y activa como profesional, preocupada y cariñosa como madre. Osvaldo es el mayor de dos hermanos: Jorge, su hermano menor, que nos acompaña hoy con su familia, también es cirujano, destacado miembro de esta Sociedad. El se ha desempeñado profesionalmente hasta hoy en la ciudad de Talca, ha compartido especialidad con Osvaldo y ha contribuido activamente al desarrollo de ella en esa ciudad 
incluyendo la creación de un Centro de Diagnóstico Endoscópico de Cáncer Gástrico.

Osvaldo fue alumno del Instituto de Humanidades Luis Campino desde primera preparatoria. No quiero herir su modestia, pero fue un alumno modelo, siempre de primer lugar y muy querido por sus compañeros. Además era aficionado al deporte. Me he enterado que practicó formalmente esgrima, natación y también, no sin sorpresa de mi parte, practicó básquetbol y además fútbol. Su excelente desempeño determinó que fuese adelantado un año de curso, por lo que ingresó, por elección propia sin tropiezos, a la Escuela de Medicina de la Universidad Católica de Chile a los 17 años de edad el año 1960.

En la Escuela Osvaldo siempre se destacó como alumno de excelencia. Fue también un alumno de actitud generosa, excelente amigo, querido por sus compañeros, entusiasta partícipe de la convivencia estudiantil propia de una época en que prácticamente todas las Facultades compartíamos el mismo campus. Osvaldo egresó el año 1966 y recibió su título al año siguiente, iniciando de inmediato su Programa de Especialización en Cirugía General, en la misma Universidad. Osvaldo completó este programa de nuevo y como siempre, con excelencia, el año 1970.

En aquellos años los vientos magallánicos trajeron desde Punta Arenas a Santiago a María Eugenia Valdés García, quien habría de ser su esposa. Quiero interrumpir por un momento el recuento curricular de Osvaldo, para incluir formalmente y hacer parte merecidamente a María Eugenia en este homenaje. Muchos de ustedes la conocieron y la recuerdan. Era una mujer extraordinaria. Si hubiera que elegir un rasgo central en ella, diría que era su sencillez. En esa sencillez se incluía también una gran calidez. Usaba estas cualidades para disimular con modestia su amplísima cultura: María Eugenia era arquitecto, paisajista, aficionada a la pintura, intérprete de inglés y francés, gran lectora, conocedora de historia y de música, además tenía una alta cultura gastronómica que disfrutábamos los que visitamos su casa siempre generosamente abierta. La Quena, como le decíamos, se fue prematura e inesperadamente un día 11 de Marzo, hace ya 3 años. Ese día dramático es imborrable para nosotros y creo que es nuestro doloroso deber recordarlo hoy con la pena que no se pasa, con una resignación difícil de lograr pero también con humildad. Nos demuestra cuán frágiles somos y nos advierte cuan cerca, cuan milimétricamente cerca, puede estar un procedimiento simple de la catástrofe. Sin embargo, quiero pensar también que la presencia de alguien tiene sin duda una dimensión distinta que viene del espíritu. Su ausencia física se ha transformado desde entonces en una presencia espiritual permanente para Osvaldo y para todos quienes la conocimos.
Osvaldo construyó su vida formando con la Quena una familia de unidad ejemplar: nos acompaña Osvaldo Llanos Valdés, fruto de este matrimonio, médico, intensivista, casado con Alejandra Rodríguez, también colega nuestra, quienes le dieron a Osvaldo maestro y a M. Eugenia quizás la mayor de sus felicidades: sus seis nietos. Entre ellos está Osvaldo Llanos Rodríguez, que constituye la cuarta generación de "Osvaldos Llanos", y que, junto con todos sus hermanos, nos augura un futuro de mucha esperanza. En esos nietos fue donde ambos literalmente encarnaron de manera simple pero incondicional su capacidad de querer, y aun cuando este homenaje es sobre un maestro de la cirugía, fue en ese ejemplo de abuelos donde muchos encontramos lo mejor de los rasgos de ambos.

Después de la beca primaria Osvaldo se desempeñó por tres años en el Servicio de Cirugía y en el Servicio de Urgencia del Hospital de Talca. Esos fueron años de abundante y variado trabajo quirúrgico, que sin duda contribuye a consolidar la formación de un cirujano. En aquellos años el trabajo no tenía el límite estricto de una especialidad de modo que se ejercía como cirujano general incluyendo cirugía digestiva, cirugía vascular, cirugía tiroidea y mamaria, entre otras, además de la urgencia. Coincidimos con Osvaldo en Talca y doy testimonio del valor del compañero generoso, con quien compartimos largas horas de pabellón y también de convivencia familiar. A veces, como decíamos entonces que por "confluencias de los astros", pasábamos dos o tres días continuos de actividad quirúrgica que eran posibles en buena medida gracias a nuestra juventud. Ya en ese entonces se manifestó la vocación académica y docente de Osvaldo al ejercer la responsabilidad de Secretario Ejecutivo de la Regionalización Docente Asistencial Universidad Católica-VII Zona de Salud. Este vínculo se mantiene hasta hoy con evidente beneficio para ambas partes.

En 1973, Osvaldo fue llamado a Santiago desde la Universidad Católica, iniciando así su larga y fructífera trayectoria académica cuya extensión y variedad la hace muy difícil de resumir. En síntesis, entre 1973 y 1979 fue instructor asociado, luego Profesor Auxiliar hasta 1981, luego Profesor Adjunto y finalmente a partir de 1989, fue promovido a Profesor Titular de Cirugía, a la edad de 46 años. Paralelamente, Osvaldo se desempeñó por dos años, entre 1973 y 1975 como Jefe de Turno del Servicio de Urgencia del Hospital Militar de Santiago.

Por razones propias del desarrollo institucional, Osvaldo incorporó e inició la endoscopia digestiva alta, de fibra óptica en nuestro hospital. Esta fue una decisión trascendente y visionaria porque permitió incorporar definitivamente este 
importantísimo recurso diagnóstico y terapéutico al quehacer quirúrgico de nuestra especialidad. Hoy se ha consolidado como una actividad compartida en equipo con nuestros gastroenterólogos, lo que ha enriquecido y ampliado nuestra labor asistencial y docente generando un modelo de trabajo claramente ventajoso. Puedo mencionar que la primera polipectomía endoscópica gástrica de nuestro país la comunicó Osvaldo en la Sociedad Chilena de Gastroenterología en 1974.

Buscando ampliar sus horizontes, Osvaldo fue becado por dos meses en Japón, en el Seminario de Cáncer Gástrico, en 1974. Sin embargo, su formación científica como cirujano académico se consolidó sin duda entre 1975 y 1977 cuando se desempeñó en el Departamento de Cirugía de la Universidad de Texas Medical Branch, con el patrocinio del NIH y la Fogarty International Fellowship. Su mentor fue el Dr. James Thompson, miembro honorario de nuestra Sociedad en Chile, quien fue un destacadísimo investigador y cirujano llegando a la Presidencia del American College of Surgeons. El Dr. Thompson desarrolló vínculos de amistad personal con Osvaldo y María Eugenia que fueron permanentes desde entonces. Además, personalmente me manifestó su admiración por la personalidad recta y sobria del nuevo maestro y por su impresionante capacidad de trabajo. Durante ese período surgieron numerosas publicaciones científicas de alto nivel, con especial dedicación al campo de las hormonas digestivas. Su estadía en ese centro le permitió posteriormente validar, por primera vez en 1978, el Radio-Inmuno ensayo de Gastrina en Chile, lo que constituyó un hito para el estudio y diagnóstico de algunos tumores neuroendocrinos entre otras patologías de la especialidad.

Dentro de sus múltiples intereses como cirujano, está el campo de la Hipertensión Portal. Desde temprano se interesó por esta patología, pero de nuevo respondiendo a sus inquietudes de perfeccionamiento, fue al departamento de Cirugía de la Emory University, en Atlanta y a Pittsburgh, por dos meses, vinculándose con los Dres. Warren y Starzl, nombres asociados al más alto nivel quirúrgico de la especialidad en ese país y el mundo. A su vuelta Osvaldo concretó una serie de más de 35 pacientes cuidadosamente seleccionados que fueron sometidos a una Anastomosis Espleno Renal Distal o Shunt de Warren sin mortalidad y excelentes resultados alejados. La disponibilidad de nuevas alternativas terapéuticas ha restringido las indicaciones de esta operación cuya vigencia, sin embargo, se mantiene hasta hoy.

Osvaldo lideró además distintos proyectos de investigación con financiamiento formal de la Dirección de Investigación de la Universidad Católica y participó como coinvestigador en otros con financiamiento obtenido a través de Fondecyt.

A la labor quirúrgica, a la labor docente y a sus proyectos de investigación, Osvaldo agrega una serie de importantes responsabilidades académicas y administrativas; son largas de enumerar y sorprendentes por lo variadas. Mencionaré solo algunas:

- Jefe de Laboratorio de Cirugía Experimental.

- Jefe del Servicio de Urgencia.

- Presidente del Consejo Técnico del Hospital Clínico.

- Miembro del Consejo Económico de la Facultad de Medicina.

- Miembro del Consejo de Facultad.

- Miembro de la Comisión de Graduados.

- Subdirector de Graduados, Escuela de Medicina.

- Jefe del Servicio de Cirugía.

- Entre 1979 y 1983, con reincidencia entre 1986 y 1989 fue Director del Hospital Clínico y Dependencias Docente Asistenciales.

- Entre 1996 y 2005, Director Médico, Clínica de la Universidad Católica.

- Director Médico del Centro del Cáncer (20082010).

Su participación en los Congresos de esta y otras Sociedades ha sido permanente y generosa: es autor o co-autor de más de 25 publicaciones internacionales, de más de 110 en nuestro país y completa más de 260 participaciones en Congresos, Cursos y Mesas Redondas. Desde luego, es miembro titular de La Sociedad de Cirujanos de Chile, de la Sociedad Chilena de Gastroenterología, de la Sociedad Internacional de Cirugía, Tesorero, Secretario, Vice-presidente y Presidente del Capítulo Chileno del American College of Surgeons, Presidente de la Sociedad Médica del Centro y miembro de la European Digestive Surgery Society.

Entre sus premios, está el de ser miembro honorario, emérito y desde ahora formalmente Maestro de la Sociedad de Cirujanos de Chile y Miembro Honorario del Capítulo Chileno del American College of Surgeons.

El año 2009 recibió el Premio a la Trayectoria Académica de la Facultad de Medicina de la Universidad Católica y el 2012 se incorpora como Miembro Honorario a la Academia de Medicina del Instituto de Chile.

He querido dejar para este momento el recuento de una de las actividades más trascendentes y reconocidas de Osvaldo y creo que probablemente de las más queridas por él: Entre 1992 y el año 2006, es decir, por 14 años, Osvaldo fue el Jefe de Programa de Post Título de Cirugía General. Este cargo lo transformó prácticamente en el padre de los becados de cirugía, lo que hace que hoy tenga 
discípulos en todo el país y en el extranjero, algunos de ellos presentes hoy. Doy fe que Osvaldo está siempre presente en el cariño de todos ellos. Además, este cargo permitió que se revelaran fuertemente los rasgos del carácter de Osvaldo. El es en primer lugar un ejemplo de integridad. Cada vez que alguien lo buscaba y lo requiriera encontraba en Osvaldo una opinión clara, encontraba acogimiento y comprensión, aun en situaciones adversas. Osvaldo era exigente y ponía su límite en lo máximo y se resistía a restringir o a mutilar la docencia, en la persecución de objetivos de conocimiento muy acotados. La fuerza de su opinión podía engañosamente sugerir rigidez pero no era así por cuanto aprovechaba e incluso disfrutaba con la diversidad y con los nuevos talentos. Había tolerancia para el error pero estrictez si detrás de él se veía dejación, descuido o poco compromiso. Siempre quedó muy claro que la razón de su espíritu exigente era para beneficio de los propios becados. Estos rasgos de su personalidad fuerte pueden haberle generado muy ocasionalmente rechazo, lo que no le impedía decir la verdad, y expresar claramente su pensamiento siempre por un fin superior, nunca egoísta. Esconder lo que a su parecer no es bueno no es parte de él. Esta característica o capacidad con frecuencia se sacrifica hoy por razones de orden político, lo que para Osvaldo no es aceptable. Sin embargo, de nuevo, en este cargo tan importante, como en toda su vida, la cirugía y la docencia tenían otra faceta. Osvaldo se dejaba espacio para acoger a sus discípulos en su casa junto con María Eugenia en tardes de grata y sencilla convivencia complementadas necesariamente por la excelente gastronomía de la Quena lo que generó en los becados, admiración y agradecimiento que muchos me han reconocido hasta el día de hoy.

En la enumeración de todos los cargos y responsabilidades ejercidos por Osvaldo omití algunas porque se vinculan directamente con su labor docente del postgrado y que de alguna manera expresan la consecuencia con sus compromisos en este campo: aparte de variados comités editoriales, el año 1995 Osvaldo se integró al Directorio de Conacem, siendo luego su Vicepresidente entre el 2003 y el 2005, y su Presidente hasta el 2008, con la trascendencia que esta responsabilidad tiene en el desarrollo de la especialización médica en nuestro país. En representación de ASOFAMECH, desde el año 2008 es Vicepresidente de APICE (Agencia Acreditadora de Programas de Posgrado, de Especialidad en Medicina y de Centros Formadores de Especialistas Médicos). Además fue presidente del Comité de Formación Médica y Perfeccionamiento del Capítulo Chileno del American College. En representación de la Facultad de Medicina, integra la Comisión
Nacional de Programas y centros Formadores de Especialistas Médicos de ASOFAMECH.

Estamos frente a un currículum de extraordinaria solidez, pero como hemos visto, a Osvaldo la Medicina le ha dejado espacio para otros intereses. Aparte de sus aficiones deportivas que se han mantenido hasta hoy, me quiero referir a un aspecto que no he mencionado: Osvaldo tiene un profundo amor por el arte de la Música y desde mucho tiempo practica con constancia la ejecución del piano, donde por años ha tomado clases. Incluso pude asistir a una velada musical en su casa donde Osvaldo era el pianista. Esta afición y esta capacidad ha sido heredada también por su hijo, y con la colaboración de Alejandra su nuera, también por sus nietos que desde temprana edad y formalmente practican piano y violín. Osvaldo además se ha referido a este tema en publicaciones y en conferencias, combinando su interés por el mundo de la música con su interés por la Historia, especialmente la de la medicina.

Creo que en estos tiempos de medicina basada en evidencia, he presentado la suficiente como para avalar sobradamente esta nominación. Quisiera hacer una breve reflexión acerca de la naturaleza de este premio: este es probablemente el reconocimiento más significativo y hermoso que puede recibir un cirujano: lo otorgan sus pares de todo el país, a través de un comité independiente de alto nivel de la Sociedad de Cirujanos de Chile. A este premio no se postula ni se es postulado. Es sorpresivo y se otorga fundamentalmente por los méritos de una trayectoria personal y profesional.

¿A quién honra entonces este nombramiento? Sin duda y en primer lugar al propio nominado. Supo, como dice la escritura, hacer uso y multiplicar sus múltiples talentos para bien de los demás. Honra además a su familia, la que está aquí físicamente y a aquella que lo acompaña permanentemente en el espíritu: simplemente comparten los méritos de Osvaldo. Honra a la institución en que trabaja. Ella ha sido la que puso el terreno para su trabajo y la primera en beneficiarse de él. Honra también, sin duda, a sus amigos, especialmente al que rinde este homenaje, por lo mucho que hemos aprendido de su generoso ejemplo. También honra a los que han sido sus discípulos: pueden enorgullecerse de haber conocido y aprendido de este profesor ejemplar. Finalmente, honra a la propia Sociedad de Cirujanos de Chile que ha sabido reconocerlo y que ahora lo incorpora en propiedad a su galería de maestros. Los invito a saludar a Osvaldo con un fuerte aplauso.

Gracias.

Dr. Sergio Guzmán B. Profesor Titular de Cirugía Universidad Católica de Chile 


\section{Discurso del Maestro}

Nada más sorpresivo para mí fue el anuncio de esta máxima distinción de la Sociedad. Si uno mira para atrás, a lo mejor podría encontrar, con humildad, algún argumento positivo para ello, quizás en el sentido en que enfoqué mi vida profesional y académica. En todo caso recibo con modesto orgullo este premio que me permite compartir la galería de muy distinguidos maestros de nuestra sociedad.

Agradezco a la Sociedad de Cirujanos de Chile esta honrosa distinción, la Sociedad tiene una trayectoria destacada tanto en el progreso de nuestra especialidad, como en el desarrollo profesional de los cirujanos. Creo haber colaborado con nuestra organización y haber participado en todas las tareas que se me ha encomendado.

Los agradecimientos son muchos. Primero a Dios que me mostró el camino de desarrollo como persona, como médico y como cirujano en la vida. Debo decir sin pedantería, sinceramente, que si bien el camino ha sido arduo, no lo he sentido de mayor dificultad y los pasos se me fueron dando en forma fluida, sin duda guiados por un ser superior.

Los agradecimientos a mi familia. A mis padres quienes siempre nos inculcaron los principios de vida, estudio y espíritu de superación para ser hombres de bien y útiles a la sociedad. No sé con seguridad quién o qué ejemplo me llevó a estudiar medicina. No había médicos en mi familia. A posteriori, en retrospectiva, he sospechado que pudo haber sido mi pediatra, personaje que me impresionaba por su presencia, su imponencia, seriedad y gravedad de su accionar. A la vez de su gentileza y bondad en el trato. Muchos años más tarde, al hacer mi internado de pediatría, encontré una placa en su honor en el hospital Roberto del Río.

Agradecimientos a mi familia, a mi señora, María Eugenia, hoy físicamente ausente, extraordinaria mujer, por su amor y comprensión, generosidad y paciencia, su sabiduría y su ejemplo de vida. A ella dedico en forma especial este premio. A mi hijo y a mi nuera, quienes me han dado 6 hermosos nietos y me han ayudado a sobrellevar la pena de esta etapa de mi vida. A todos mis familiares más cercanos, a mis grandes y buenos amigos que se han incorporado de corazón a mi familia.

Agradezco a todos mis maestros y profesores, desde mi colegio, el Instituto de Humanidades Luis Campino, hasta la universidad. Mi vida ha sido moldeada por muchos maestros. No los mencionaré por el riesgo de omitir a algunos. En la Escuela de Medicina a mis docentes de ciencias básicas quienes nos transmitieron la inquietud y el pensamiento científico. En la clínica a distinguidos docentes quienes nos enseñaron a tratar al enfermo, escucharlo, examinarlo y entregarle apoyo y confianza. En cirugía, faltando a mi propósito de no nombrarlos, sin desmerecer a otros, quiero destacar a don Arnaldo Marsano, nuestro verdadero maestro de generaciones, eximio profesor por su pensamiento clínico y sabio criterio, su humildad, su disponibilidad, su generosa paciencia y experiencia en enseñarnos, enseñarnos "de la mano", tal cual, las destrezas y habilidades quirúrgicas en el pabellón. Todo esto con un profundo respeto por el paciente que se entrega confiado a su cirujano.

Entre mis maestros extranjeros, quisiera destacar al doctor James C. Thompson, en cuyo departamento de cirugía de la universidad de Texas tuve el privilegio de ser becado por la NIH. El doctor Thompson, quien tenía un gran laboratorio de investigación en su departamento de cirugía, me enseñó, entre otras cosas, la importancia de la inquietud científica y de la rigurosidad y disciplina en la investigación y en la proyección clínica de los nuevos conocimientos para el mejor estudio y tratamiento de los enfermos.

Como he dicho, creo que no cometo pecado de omisión al no nombrar a otros, numerosos y desinteresados maestros que hemos tenido en nuestra formación.

Es necesario reconocer a mi universidad y a mi escuela de medicina. Cuando entré a estudiar medicina escogí libremente a la Universidad Católica. En ese entonces, nuestra escuela era pequeña, emergente, con apenas 30 años de vida, con pocos alumnos, muchas imperfecciones, pero con un grupo selecto de profesores quienes con esfuerzo, imponían la mística de sacar adelante ese proyecto por sobre todas las dificultades y limitaciones. Creo que esta mística se transmitía a nosotros, los alumnos. Me tocó la suerte de asistir, en cincuenta años, al proceso de superar la etapa de escuela emergente hasta llegar a constituir lo que es hoy día, una de las mejores del país y con atractivos incluso para otros países. Tuve la suerte de asistir a este magnífico progreso, siempre en evolución, y de colaborar modestamente con un granito de arena.

Agradezco a nuestra universidad, a nuestra facultad y a nuestra escuela de medicina el haberme dado la oportunidad de desarrollarme como médico, como académico y como persona. Creo que sinceramente encontré en ella el estímulo, la fuerza y el empuje para tratar de ser un buen profesional, un buen académico, un buen doctor. Como se sabe, las instituciones las forman y sostienen las personas y la calidad de una universidad está dada por el actuar de la suma de personas que estudian en ella, que trabajan en ella, que piensan en ella, que creen en ella y por eso actúan con convicción y pasión por ella, tanto en la búsqueda de la verdad como en el aprender a servir al próximo. 
A todos los aquí presentes nos ha tocado vivir cambios trascendentes en la medicina y por ende en la cirugía. Largo sería de enumerar los diferentes avances que compiten por ocupar los primeros lugares entre ellos, tanto en mayores conocimientos como en progresos tecnológicos. Debemos entender que el cambio, hoy muy englobado en el concepto de innovación, es un fenómeno continuo, normal, natural, imparable e insoslayable. En todas las épocas ha habido cambios en la historia de la medicina, sólo que ahora parecen ser más rápidos. Está demás mencionar como ejemplo, lo trascendente que fue para la cirugía el comienzo del uso de la anestesia y de la antisepsia a mediados del siglo XIX. Que el cambio es un fenómeno ineludible lo ilustró Heráclito, filósofo griego, al decir que ningún hombre cruza dos veces el mismo río, porque la segunda vez ya no es el mismo río, y él ya no es el mismo hombre.

Es motivo de atención, que los cambios actuales se pueden ver en dos grandes aspectos: uno, en los aspectos científicos y tecnológicos, cuya utilidad es indiscutible y en segundo lugar en las relaciones médico pacientes y en la manera en que hoy se ejerce la medicina. En este segundo aspecto es donde a veces se ven algunos cambios negativos y con alguna frecuencia escuchamos hablar de la deshumanización de la medicina actual, que podrían significar cambios en la vocación e interés de los médicos. Debemos preocuparnos de ello y trabajar para corregir los errores. Los cambios en la medicina no son sino, de algún modo, reflejo de los cambios en la sociedad, en la cultura y en la civilización. Así y todo me parece casi increíble que se haya tenido que elaborar una especie de código sobre los derechos y deberes de los pacientes, tema que me parece obvio y que debiera estar en el pensamiento de todo médico.

Uno de los objetivos de mi quehacer en la universidad ha sido la formación de los cirujanos. Para ello es necesario revisar con frecuencia cuáles son las características esperables en un cirujano, más allá de los aspectos científico técnicos propiamente tales. Repito, el cirujano debe poseer conocimientos y habilidades, destrezas propias de la especialidad. A ellos debe agregarse el criterio clínico. Debe tener inquietud profesional y científica, capacidad de estudio permanente, capacidad de trabajo y dedicación a los pacientes, honestidad, integridad y honor, humildad -el cirujano no puede o no debe ser soberbio-, compasión, valentía y mesura, espíritu de servicio, compromiso con sus enfermos, paciencia, amabilidad en el trato y respeto por los demás. Debe poseer capacidad de decisión, conciencia de sus limitaciones, saber detenerse a tiempo y pedir ayuda cuando sea necesario. Es recomendable como en todas las actividades de mayor repercusión social, disciplina y sobriedad en el vivir, como ya lo dijo Paracelso en el siglo XVI, en fin, son muchas las cualidades del cirujano descritas por diferentes autores pero la mayoría giran en torno a las que he mencionado.

Quisiera agregar que junto con compartir su tiempo de trabajo con el dedicado a la familia, a la que no se debe descuidar, es bueno, es útil, que el cirujano dedique parte de su atención a las humanidades: el arte, la literatura, la pintura, la música, la disciplina que más le atraiga y en el grado que buenamente pueda, desde la observación o la audición, hasta la ejecución en distintos grados. Es bueno para entender mejor al mundo y a los seres humanos, motivos de nuestro quehacer. Se ha dicho que los cirujanos, al estar enfrentados a diario con la enfermedad, con el sufrimiento y con la muerte, quizás necesitemos más que otras personas de los efectos restauradores y benéficos que entrega el arte en cualquiera de sus manifestaciones. El arte es un alimento para el espíritu. Por otra parte, no hay que olvidar que la medicina además de una ciencia es un arte, en especial la cirugía. Es un arte saber cultivar las relaciones humanas y aplicar los conocimientos y destrezas que debe dominar el cirujano al tratar a su paciente. Al respecto Sinclair Lewis, premio Pulitzer en 1925 y Nobel en 1930, en su novela Arrowsmith, dice que hay 3 libros que todo médico debiera estudiar: la anatomía de Gray, la Biblia y Shakespeare (cienciareligión y humanidades). Además quisiera recordar que el arte estimula la creatividad, virtud de alguna manera también necesaria para el cirujano.

Pasando a otro aspecto, quisiera también agradecer a mis pacientes, quienes a lo largo de más de 45 años me han permitido colaborar en el alivio de su dolor, con la confianza que han depositado en $\mathrm{mi}$ persona.

Una mención especial para mis alumnos a lo largo de estos años. Su realización y éxito son motivo de satisfacción por haberme permitido colaborar en su formación. La docencia significa no sólo transmitir conocimientos y experiencia, guiar sus primeros pasos, facilitar un desarrollo individual y colectivo, en la búsqueda de la excelencia, que hace necesario tener como objetivo que los alumnos superen a los profesores. A su vez, al igual que todos ustedes, yo he aprendido mucho de mis propios discípulos. Ser maestro significa tener fe en el individuo, fe en nuestros alumnos, fe en la primacía de la riqueza intelectual.

A mis compañeros de trabajo, tengo la fortuna de formar parte de un grupo de colegas muy destacado por su capacidad, por su unión y convicción de lo que significa trabajar en un verdadero equipo, cohesionado en la búsqueda de fines comunes: atender a nuestros enfermos de la mejor manera posible, con parámetros objetivos de evaluación, para enseñar buscando la excelencia, como otros lo hicieron con 
nosotros, para cuidar y elevar el nivel de nuestra cirugía y de nuestra institución, para cuidarnos los unos a los otros. No es tarea fácil lograr estas metas, pero con dedicación y comprensión, el fruto de años de trabajo está a la vista.

El trabajo de un maestro debe contemplar el saber respetar a los demás, a los enfermos y a su familia, a los colegas, a las personas de colaboración, enfermeras, auxiliares y a todos quienes nos ayudan a desempeñar nuestra misión. Saber agradecer y saber corregir con respeto y con cariño engrandece.
He dejado para el final, muy importante, el reconocimiento a mi gran amigo, el doctor Sergio Guzmán, compañero de toda una vida quirúrgica, la que labró una verdadera amistad familiar, de quien aprendí muchas virtudes, con quien compartimos muchas satisfacciones por las realizaciones y logros, pero también muchas horas de trabajos, desvelos y vicisitudes, que nos templaron el espíritu, Maestro de la cirugía chilena, quien tuvo la gentileza de realizar este inmerecido homenaje a mi persona.

Muchas gracias. 